\title{
A new scheme for analysis of pore characteristics using centrifuge driven non-toxic metal intrusion
}

\author{
Shu Jian Chen $\cdot$ Yuan Tian $\cdot$ Chen Yang Li • \\ Wen Hui Duan
}

Received: 4 March 2016/Accepted: 18 May 2016/Published online: 28 May 2016

(C) Springer International Publishing Switzerland 2016

\begin{abstract}
This study investigates the intrusion behavior of a non-toxic low-melting-point metal using centrifugation to develop a better pore characterization technique for cementitious materials such as ordinary Portland cement. This study suggests that centrifugation is a safe and effective method for driving melted low-melting-point alloys into cementitious material at pressure between 12 and $15 \mathrm{MPa}$. The non-toxic Field's metal alloy is suggested as a replacement for the toxic Wood's metal. For pores between $100 \mathrm{~nm}$ and $10 \mu \mathrm{m}$, Field's metal can be used without vacuuming the sample before intrusion. For pores $>10 \mu \mathrm{m}$, pre-vacuuming of the sample is suggested to prevent the extrusion of hardened Field's metal from large pores. Different failure modes for the extrusion phenomenon were analyzed and the results suggest that weak bonding between the Field's metal and the cement matrix is the main cause for the extrusion. The new scheme may also be applicable to porous geomechanical materials such as porous rock, sand and clay.
\end{abstract}

Electronic supplementary material The online version of this article (doi:10.1007/s40948-016-0029-z) contains supplementary material, which is available to authorized users.

S. J. Chen · Y. Tian · C. Y. Li · W. H. Duan ( $₫)$

Department of Civil Engineering, Monash University, Clayton, VIC 3800, Australia

e-mail: wenhui.duan@monash.edu
Keywords Pore characterization - Field's metal · Intrusion $\cdot$ Cement $\cdot$ BSE $\cdot$ Image analysis

\section{Introduction}

Pore characteristics determine many properties of porous cementitious materials, such as mechanical properties, transport properties, creep, and shrinkage. However, the proper method for characterization of pores is still debated. The main discrepancy lies in the characterization of capillary pores, where the traditional and most frequently adopted technique is a porosimetry method such as Mercury intrusion porosimetry (MIP). The porosimetry method has limitations, such as its inaccurate interpretation of pore size due to irregular and ink-bottle-like pores in cementitious materials (Moro and Böhni 2002). Image-based characterization is often suggested for realistic observation of pores (Abell et al. 1999). For image analysis, the pores needs to be filled with a support material such as epoxy resin (Kjellsen et al. 2003) and then ground and polished to expose a flat cross-section of the material to allow imaging of the pores. The main limitation of that method is that epoxy resins have low contrast with the cementitious matrix, making it difficult to obtain clear pore images.

In the literature, the best solution for characterizing capillary pores is backscattered electro-microscope (BSE) imaging using Wood's metal as the intrusion 
material (Diamond 2000; Nemati 2000; Stroeven et al. 2010) to fill the pores. Wood's metal has been melted at temperatures above $71{ }^{\circ} \mathrm{C}$, driven into the pores with pressure, and cooled to harden in the pores (Lange et al. 1994; Nemati 2000). The use of Wood's metal to replace epoxy can significantly improve the contrast and resolution of pores under BSE imaging because of the large difference between the atomic number (Lloyd 1987) of the metal and that of cement (Lange et al. 1994; Nemati 2000).

Although the superiority of the Wood's metal intrusion has been known for decades, its application is very limited. There are extensive publications reporting the use of MIP (Kumar and Bhattacharjee 2003) and epoxy impregnation techniques (Wong et al. 2006), but only a handful of studies (Dullien 1981; Lange et al. 1994; Diamond 2000; Nemati 2000; Stroeven et al. 2010) have used the metal intrusion/ impregnation technique. The major limitations of the Wood's metal intrusion technique include: (1) the requirement for customized high-pressure chambers and sample holder (Willis et al. 1998) to sustain pressure; (2) the difficulty of controlling the pressure, such that customized control units (Lloyd et al. 2009) have been needed; (3) the high toxicity of Wood's metal due to its $\mathrm{Pb}$ and $\mathrm{Cd}$ content (Chen et al. 1975; Schubauer-Berigan et al. 1993), creating high risk for those involved in the regular handling (grinding and polishing) of samples.

This study investigates the use of an alternative intrusion method and intrusion material to address the problems of Wood's metal intrusion and to provide a better characterization method for the field. There are two key innovations in this study. Firstly, the intrusion performance of a non-toxic, low-melting-point alloy, namely Field's metal, is investigated for the first time. Secondly, a new centrifugation-driven method is invented so that pressure can be applied and controlled using standard laboratory centrifuges. This new scheme is called centrifugation-driven low-meltingpoint metal intrusion (CLMI) and requires no custombuilt control units. In addition, the effect of vacuum environment on the intrusion process is also investigated. Porosity are also critical for the mechanical properties and permeability of geomechanical materials such as sandstone, dolomite, sand and clay. The proposed method can be used to provide new insight into the pore structure of these geomechanical materials.

\section{Experimental program}

\subsection{Materials and instrumentation}

Type GP ordinary Portland cement, conforming to the requirements of Australian Standard AS 3972 (Standards Australia 2010), was used. Wood's metal and Field's metal were purchased from Rotometals, Inc. The relevant chemical composition and physical properties are listed in Table 1. Field's metal is proposed as an alternative low-melting-point metal for intrusion, as it is non-toxic, containing neither lead nor cadmium.

An Eppendorf Centrifuge 5702 with the swing bucket rotor A-4-38 was used. A Nova 450 SEM was used to perform BSE imaging of the sample. Two containers were designed to hold the sample and highpressure molten metal in place. Assembly of the container is shown in Fig. 1a, b. The container was composed of three parts. Part 1 includes a tube to raise the pressure head of the molten method at the top seal of the sample chamber. Part 2 is the chamber in which the cement sample is seated. Part 3 is the bottom seal of the sample chamber. These three parts were made of high strength 2024 aluminum alloy and assembled using 6 M4 screws made of high tensile steel. The top of the tube was threaded and sealed using a short M4 screw to prevent spillage. The container could be cleaned in a hot water bath and reused.

\subsection{Sample preparation and centrifuge intrusion process}

Two mixes of ordinary Portland cement (OPC) paste and one mix of OPC mortar were cast into $20 \mathrm{~mm}$ cubes with the water to cement $(\mathrm{w} / \mathrm{c})$ ratios of 0.4 and 0.8. No additive was used in the three mixes. Specimens were demoulded after $16 \mathrm{~h}$. Then the $\mathrm{w} / \mathrm{c}=0.4$ samples and the $\mathrm{w} / \mathrm{c}=0.8$ samples were cured in saturated calcium hydroxide solution for 28 and 7 days, respectively. The cured samples were then placed in ethanol to stop hydration. The cement pastes were sectioned and trimmed into the approximate size of $5 \mathrm{~mm}$ by $5 \mathrm{~mm}$ by $5 \mathrm{~mm}$ with ethanol as the coolant and lubricant. The cement samples were reserved in vacuum for 1 week to remove liquid from the pores.

The centrifuge was used to provide the high pressure for the liquid metal to intrude into the pores of the cement samples. A schematic diagram of the calculation of intrusion pressure is presented in 
Table 1 Properties of Wood's metal and field's metal

\begin{tabular}{|c|c|c|c|c|c|}
\hline & Composition by weight & Melting point & Contact angle & Yield strength & Density \\
\hline $\begin{array}{c}\text { Wood's } \\
\text { metal }\end{array}$ & $\begin{array}{l}50 \% \text { cBi, } 26.7 \% \text { Pb, } 13.3 \% \text { Sn, } 10 \% \\
\text { Cd MatWeb }(2015 \mathrm{a}, \mathrm{b})\end{array}$ & $\begin{array}{l}70{ }^{\circ} \mathrm{C} \text { MatWeb } \\
(2015 \mathrm{a}, \mathrm{b})\end{array}$ & $\begin{array}{l}130^{\circ} * \text { Willis } \\
\text { et al. (1998) }\end{array}$ & $\begin{array}{l}\sim 60 \mathrm{MPa}(\mathrm{Kim} \\
\text { et al. 2006) }\end{array}$ & $\begin{array}{l}9.38 \mathrm{~g} / \mathrm{cm}^{3} \\
\text { MatWeb (2015a, } \\
\text { b) }\end{array}$ \\
\hline $\begin{array}{l}\text { Field's } \\
\text { metal }\end{array}$ & $\begin{array}{l}32.5 \% \mathrm{Bi}, 51 \% \text { In, } 16.5 \% \text { Sn MatWeb } \\
\quad(2015 \mathrm{a}, \mathrm{b})\end{array}$ & $\begin{array}{l}62{ }^{\circ} \mathrm{C} \text { MatWeb } \\
(2015 \mathrm{a}, \mathrm{b})\end{array}$ & $\begin{array}{l}130^{\circ} * \text { Willis } \\
\text { et al. (1998) }\end{array}$ & $\begin{array}{l}<33.4 \mathrm{MPa} \\
\text { MatWeb (2015a, } \\
\text { b) }\end{array}$ & $8 \mathrm{~g} / \mathrm{cm}^{3}$ \\
\hline
\end{tabular}

* Due to limited information on contact angle for low melting point metals, they are assume to have similar contact angle as mercury (Willis et al. 1998)

Fig. 1c, d shows the CLMI device seating in a pair of buckets on a centrifuge rotor. The intrusion pressure $\mathrm{P}$, measured in $\mathrm{MPa}$, was calculated as

$P=\frac{1}{2} \rho \omega^{2}\left(2 L_{1} H+H^{2}\right) / 10^{9}$

where $\rho$ is the density of liquid metal, with the value of $9.8 \mathrm{~g} / \mathrm{cm}^{3}$ for Wood's metal and $8 \mathrm{~g} / \mathrm{cm}^{3}$ for Field's metal, $\omega$ is the centrifuge speed measured in $\mathrm{rad} / \mathrm{s}$. $\mathrm{L}_{1}=48 \mathrm{~mm}$, is the distance from the center of the centrifuge to the surface of the liquid metal in the tube, $\mathrm{H}=82 \pm 2 \mathrm{~mm}$, is the depth of liquid metal in relation to the surface of the cement sample.

The CLMI consisted of the following steps. (1) Cement samples were seated in the chamber of the tube and the three parts of the tube were assembled with M4 screws, leaving the top open. (2) The tubes were then preheated to above $90{ }^{\circ} \mathrm{C}$ in a water bath. The liquid metal gradually filled into the tube from the top as the metal particles melted. The bath was sealed to prevent water entering the container. (3) When the depth of the metal reached $82 \pm 2 \mathrm{~mm}$, the tube was sealed. The tubes were wrapped in foam plastic and seated into the centrifuge buckets (as shown in Fig. 1d). (4) The sets were heated in the water bath to maintain the temperature above $90{ }^{\circ} \mathrm{C}$ for $5 \mathrm{~min}$ and then centrifuged. The centrifuge time was set at $10 \mathrm{~min}$ to allow complete solidification of the liquid metal during the centrifugation process. (5) The top screws of the sets were reopened and the vacant depth of the metal was refilled to the level of $82 \pm 2 \mathrm{~mm}$ (as in step 3 ) to compensate for the volume intruded into the sample. (6) Step 4 was repeated. For pre-vacuumed samples, step (2) was performed in a vacuum oven with the container filled with metal particles and then melted under the vacuum.

Four samples were intruded, as listed in Table 2. W1 and W2 are samples intruded with Wood's metal while F1, F2 and F3 are samples intruded with Field's metal. F3 is a mortar sample with quartz sand as the fine aggregate and the rest of the samples are paste sample with no sand. The centrifuge speed was set at 4400 RPM, which, based on Eq (1), provided the intrusion pressure of $15.2 \pm 0.34$ and $12.4 \pm 0.28 \mathrm{MPa}$ for Wood's metal and Field's metal respectively. The pressure for intruding the pores was set according to the well-known Washburn's equation (Diamond 2000), based on which 12-15 MPa can drive metal into pores with diameter about 100-120 nm. Pressure higher than this is not preferred since it may intrude metal into smaller pores that cannot be clearly imaged using BSE and introduce noises in the BSE images. It should be noted that the pressure difference for the two types of metals is due to their different density as shown Table 1. Although this pressure variation from 12 to $15 \mathrm{MPa}$ may slight vary the lower limit of intruded pores from 120 to $100 \mathrm{~nm}$, such difference is negligible under the BSE imaging. The centrifuge time was set at $10 \mathrm{~min}$ so that the liquid metal could completely harden during the centrifuge process. The intruded samples were removed from the chambers of the tubes and preserved in a vacuum oven and dried for $24 \mathrm{~h}$.

In comparison with the traditional external-pressure-driven methods, a unique advantage of using centrifugation as the driving force of the intrusion is that a gradient pressure can be applied on the sample. A schematic of this gradient intrusion method is demonstrated in Figure S1 (supplementary materials). Detailed instrumentation and results of the gradient intrusion will be presented in a follow-up paper.

\subsection{BSE images and image analysis}

The intruded samples were mounted in epoxy resin and then lapped and ground using silicon carbide 
Fig. 1 a Overview of container design (assembly of 3 parts), $\mathbf{b}$ the container parts before assembly, and c schematic diagram of intrusion pressure, $\mathbf{d}$ picture of the CLMI device, bucket and rotor in a centrifuge, e picture of the intruded samples

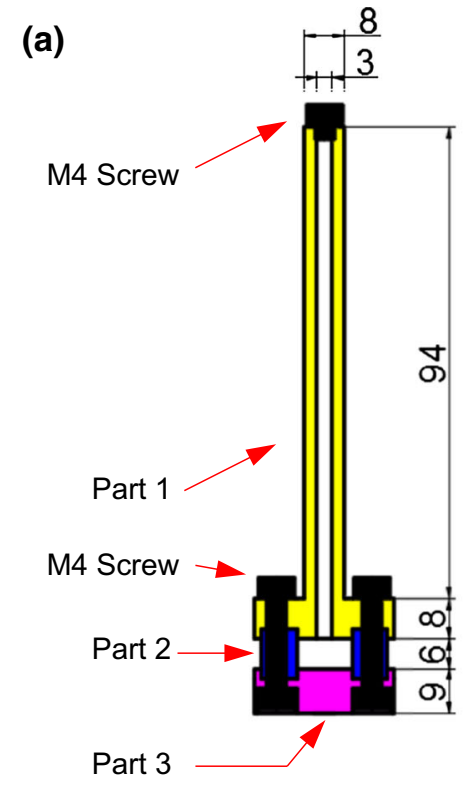

(b)

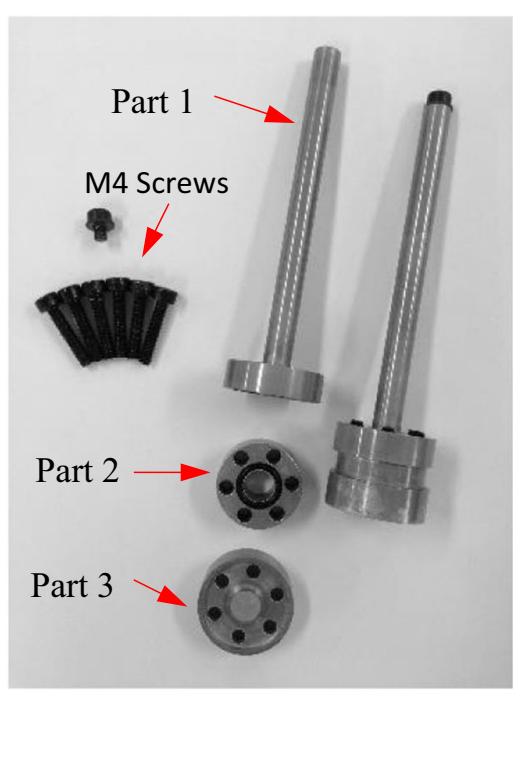

(c)
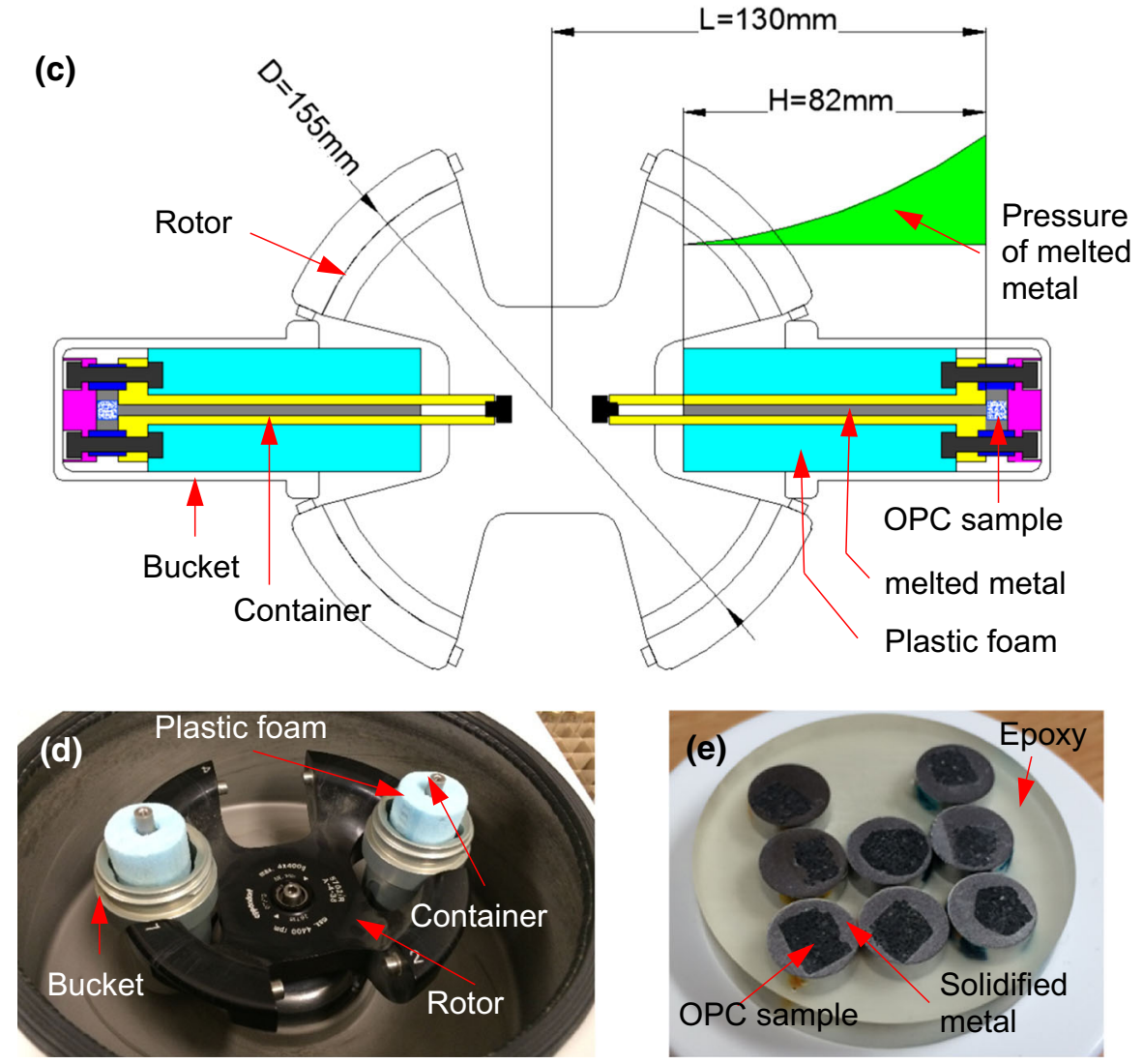

papers down to grit P1000 to expose cross-sections of the samples. The intruded samples were then subsequently polished for $30 \mathrm{~min}$ using diamond pastes in the grades of $60,25,6,1,0.25$, and $0.1 \mu \mathrm{m}$. Before moving to a finer grit, each sample was immersed into ethanol and cleaned in an ultrasonic bath remove any 
Table 2 Sample information

\begin{tabular}{llllll}
\hline Sample name & w/c & Age (days) & Sand/cement & Centrifuge speed (RPM) & Intrusion pressure (MPa) \\
\hline W1 & 0.4 & 28 & 0 & 4400 & $15.2 \pm 0.34$ \\
W2 & 0.8 & 7 & 0 & 4400 & $15.2 \pm 0.34$ \\
F1 & 0.4 & 28 & 0 & 4400 & $12.4 \pm 0.28$ \\
F2 & 0.8 & 7 & 0 & 4400 & $12.4 \pm 0.28$ \\
F3 & 0.5 & 28 & 2 & 4400 & $12.4 \pm 0.28$ \\
\hline
\end{tabular}

dirt on the surface. Figure 1e shows the polished CLMI samples which is firstly intruded and embedded with solidified metal and then mounted in epoxy. Finally, each sample was dried in a vacuum for $24 \mathrm{~h}$. Then the samples were coated with a thin layer of carbon to prevent for charging under BSE microscopy. Samples were observed and imaged under BSE microscopy at the magnification of $1000 \times$ and at the acceleration voltage of $5 \mathrm{kV}$. Five images were taken randomly at the center of each sample providing data of more than 50,000 pores on each sample.

The BSE images were further analyzed by Image-J (Ferreira and Rasband 2011). First, the scale was set based on the scale bar on the BSE image. The contrast of the image was enhanced, allowing $0.4 \%$ of the pixels to become saturated. Then the image data was converted into 8-bit grayscale, with the range of greyness 0-255. Binary images of the pores were obtained by thresholding using IsoData algorithm (Ridler and Calvard 1978). The intruded pores, identified as black pixels in the binary image, were analyzed using the inbuilt algorithm of Image-J. A scaling factor was set for each pixel based on BSE. Solid inclusions (white pixels) within pores were considered in the calculation of pore sizes. Pores at the edges of the image were ignored as their profiles were incomplete.

\section{Results and discussion}

3.1 CLMI using wood's metal and the non-toxic field's metal

Figure 2a, c show typical BSE images for CLMI for samples with different pore characteristics. These images were obtained using low-energy electrons and ultra-long electron beam dwell time ( $45 \mu \mathrm{s})$ to enhance the elemental contrast and resolution of pores, facilitating easy identification of the pores via computer-aided image analysis. A clear difference can be seen between the pores in Fig. 2a, c. Figure 2a shows the cement paste that was hydrated for 28 days with the water to cement ratio of 0.4. It can be seen that the sample possesses mainly small pores with diameter less than a few hundred $\mathrm{nm}$. More large pores are observed in the cement paste hydrated for 7 days with the water to cement ratio of 0.8 in Fig. 2c. A zoomed-in view (Fig. 2a insert) of the pores surrounding an unhydrated cement grain demonstrates the distribution of finemetal-filled pores in detail. For the large pores in the 0.8 , 7-day sample, the images (Fig. 2c insert) clearly show the curved and complex shape of pore perimeters.

As demonstrated in Fig. 2b, d, the analysis can successfully distinguish the pores (black) from the cement (white). The analysis can successfully distinguish the pores (black) from the cement (white) based on the IsoData threshold method. The threshold line is show in the greatness spectra in Fig. 2b, d, and it indicates that the cement matrix (high peaks to the left of the threshold line) can be easily separated from the pores (to the right of the threshold line). CLMI was also applied using Field's metal rather than Wood's metal. Figure 3 a shows the BSE of the Field's metal method. The Field's metal intruded samples show similar resolution and contrast to the Wood's metal intruded samples.

However, extrusion of the Field's metal is observed at some pores. As shown in the magnified view in Fig. 3b, the extruded metal seems to have been squeezed out of the pores plastically. This extrusion phenomenon of solid metal phase is an interesting finding and is discussed in detail in Sect. 3.2. For pore analysis, these areas with extruded pores can be excluded from the analysis, as shown in Fig. 3c, d. Then the cumulative pore size distribution curves ( $F$ curve) can be obtained using the same protocol as for Wood's metal. The $F$ curves for the Field's metal intruded samples are shown and compared with the Wood's metal intruded samples in Fig. 4. 
Fig. 2 Typical BSE images of Wood's metal intruded samples: a sample W1; a-insert, magnified BSE image of pores near an unhydrated cement grain; c sample W2; c-insert, magnified BSE image of large metal-filled pores. Typical binary images of pores (black) in b sample $\mathrm{W} 1$ and d sample W2. The inserts in $\mathbf{b}, \mathbf{d}$ show the greyness spectra and the threshold value of the greyness (0-255) above which are the pores (percentage indicates the fraction of pixels above the threshold)
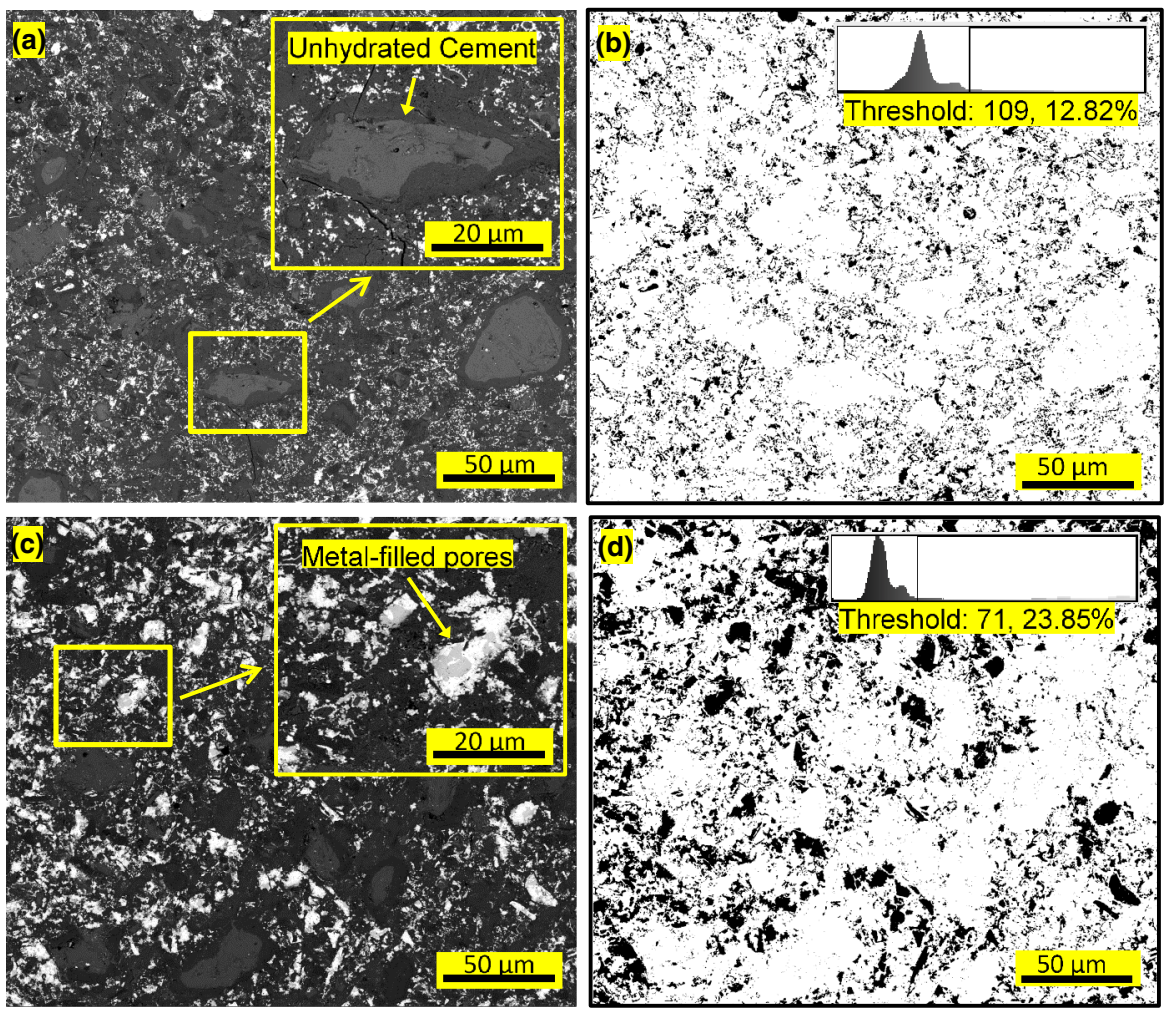

Figure 2a shows that for the 28-day w/c $=0.4$ sample, the $F$ curve of the Field's metal intruded sample overlaps with that of the Wood's metal intruded sample. The observable range of the pore diameter is from around 0.1 to $10 \mu \mathrm{m}$, about two orders of magnitude. In the 7-day sample with w/c $=0.8$, the $F$ curve of the Field's metal intruded sample overlaps with that of the Woods' metal intruded sample when $d_{p}$ is smaller than around $9 \mu \mathrm{m}$. When $d_{p}$ is $>9 \mu \mathrm{m}$, the Field's metal intruded samples display lower porosity. This is due to the exclusion of the extruded area, as the extrusion phenomenon is found to affect mainly large pores, as shown in $4 \mathrm{a}$. The higher intrusion pressure of the Wood's metal (15.2 MPa) does not result in an increase in the observable small pores. This indicates that pressure around $12 \mathrm{MPa}$ is sufficient for filling pores for BSE imaging. Excessive pressure should be avoided as it may also cause damage to the pore structure.

In general, the results show that Field's metal is a superior replacement for the toxic Wood's metal if pores with $d_{p}<10 \mu \mathrm{m}$ are of interest. For observation of large pores, Field's metal can also be used with a pre-vacuum approach to eliminate the extrusion effect, to be discussed in Sect. 3.2.

\subsection{Effect of vacuum on the intrusion process}

There are two possible causes for the extrusion observed: one is the pressure of gas trapped inside the pores; the other is the shrinkage of the matrix due to drying or relaxation of the stress generated during intrusion. To determine which of these is the dominant cause, the effect of pre-vacuuming on the extrusion phenomenon is investigated. Figure 5 presents typical BSE images of a pre-vacuumed mortar sample that contains a significant amount of large pores $(\mathrm{d}>10 \mu \mathrm{m})$ and it shows that extrusion can be mostly eliminated by the pre-vacuuming. However, the prevacuum technique causes some cracks, as shown in Fig. 5a, due to the loss of water at high vacuum and temperature. Besides the cracks, the method still allows a clear view of the large pores such as in the interfacial transition zones (ITZ) shown in Fig. 5a.

The pre-vacuumed samples indicate that that the gas trapped inside the pore is the dominant cause of the extrusion. For better understanding of the extrusion 

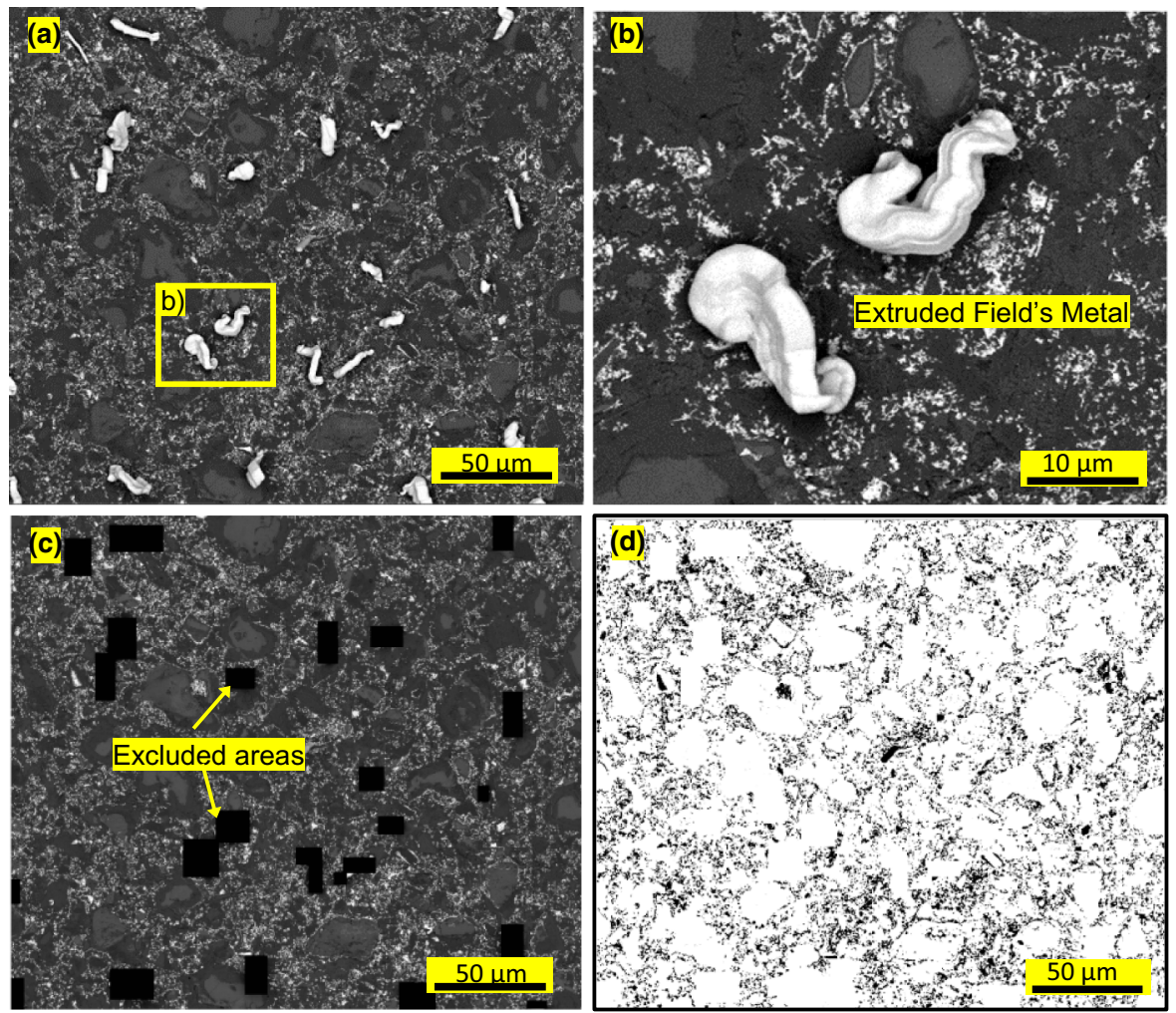

Fig. 3 a BSE images of Field's metal intruded samples, b magnified view of extrusion from large pores, $\mathbf{c}$ BSE image with the extrusion areas excluded, and $\mathbf{d}$ binary image of pores excluding the extruded area

Fig. 4 Data comparison between Wood's metal and Field's metal intrusion: a 28-day samples with $\mathrm{w} / \mathrm{c}=0.4, \mathbf{b} 7$-day samples with $w / c=0.8$

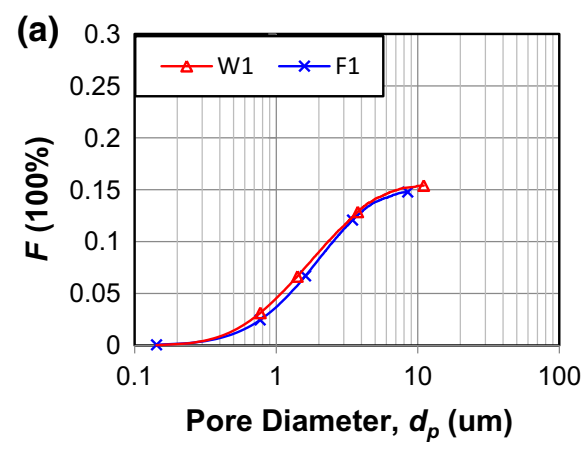

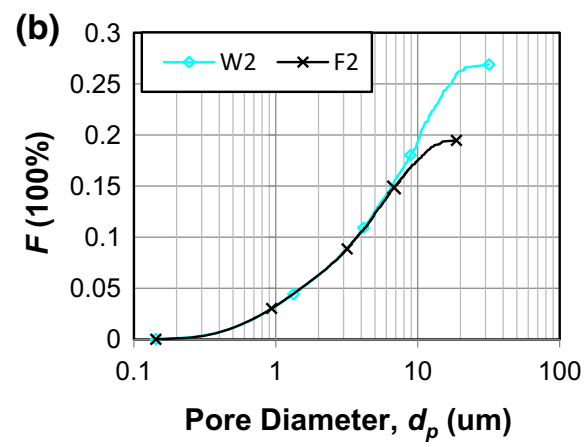

phenomenon, the mechanism is studied here. Figure 6 illustrates the extrusion process of the metal with two possible failure modes, shear failure in the metal and bonding failure between the metal and the cement matrix. Here, the intrusion considered is in atmosphere and in an ideal cylindrical pore. As shown in Fig. 3a, $\mathrm{b}$, no OPC particle is attached to the extruded metal; therefore, there is no failure in the cement matrix.
Assuming that the gas trapped at the end of the pore in Fig. 6a has a pressure of $P_{G}$, the extruding force acting at the bottom of the metal is

$N_{e x}=0.25 \pi P_{G}\left(d_{p}-2 t\right)^{2}$

where $N_{\mathrm{ex}}$ is the extruding force, $t$ is the thickness of metal attached to the cement after extrusion. If the extrusion is due to bonding failure, then $t=0$. If the 

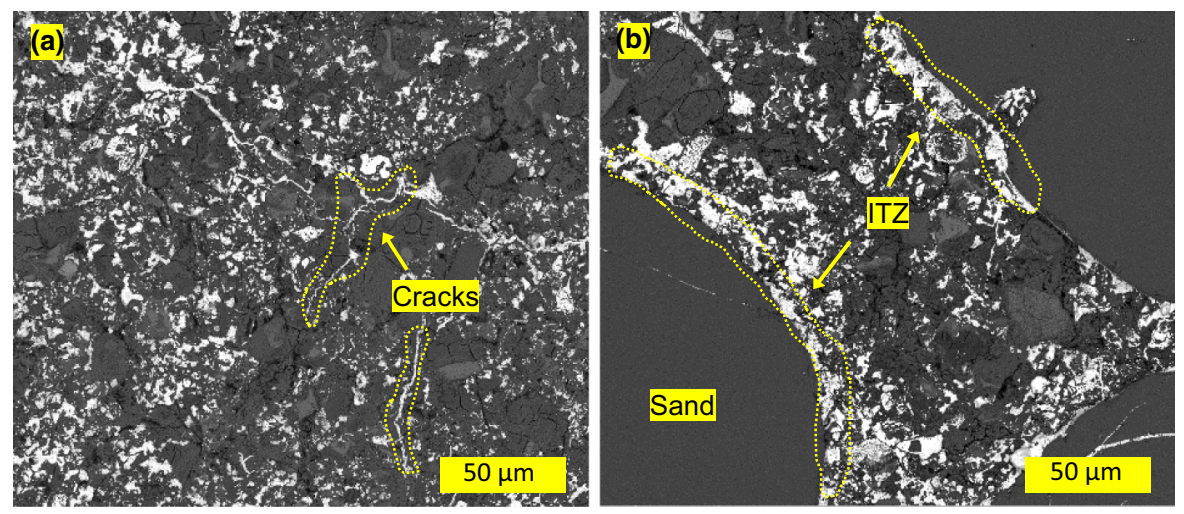

Fig. 5 BSE images of pre-vacuumed mortar samples prepared by CLMI method
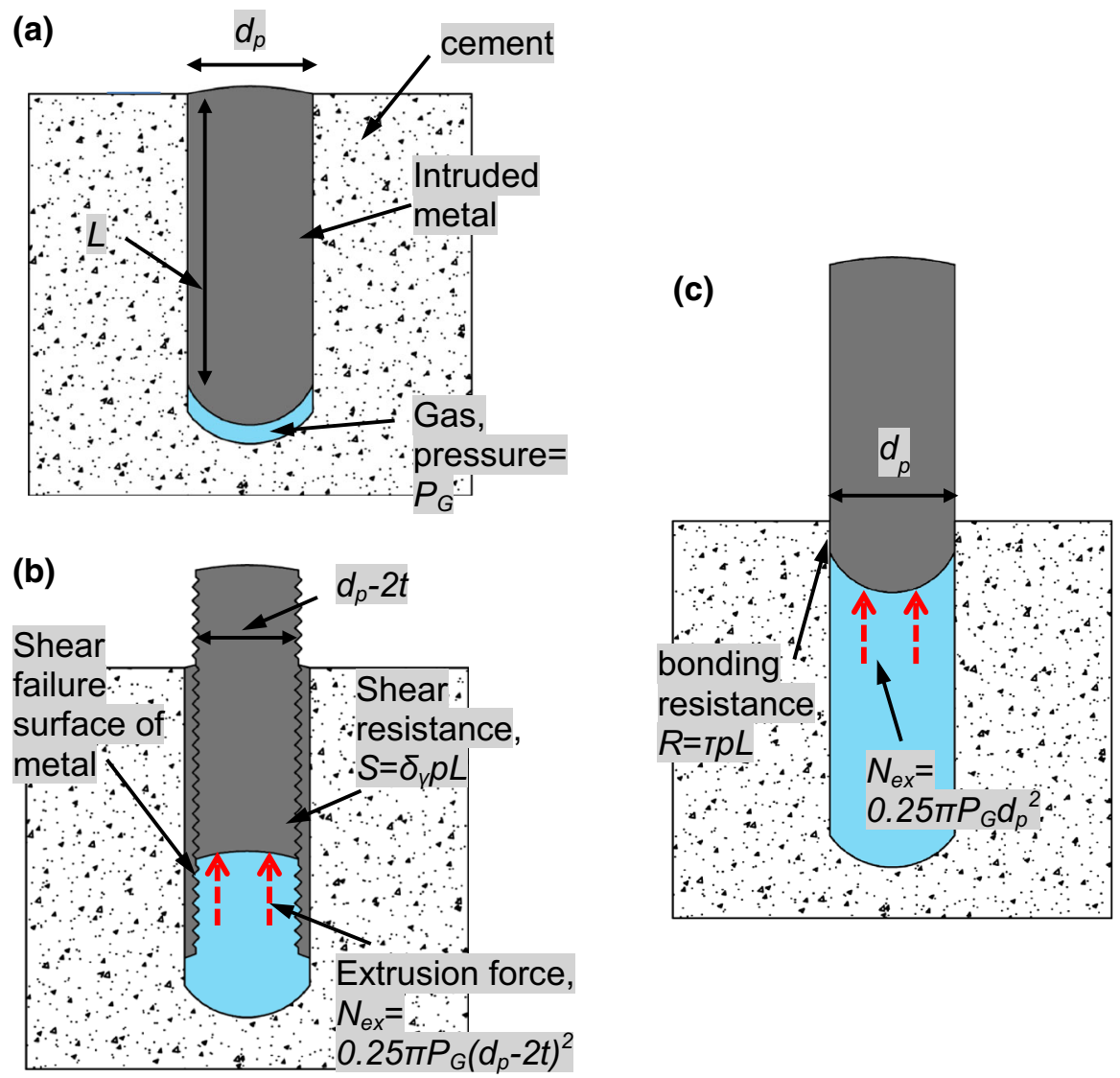

Fig. 6 Schematic of extrusion process of intruded metal in large pores: a metal in pore before extrusion, b metal extruded by shear failure of metal, and $\mathbf{c}$ metal extruded by bonding failure with cement

extrusion is due to shear failure of the metal, $t$ is a variable depending on the amount of $N_{e x}$ and the shear resistance of metal $S$, given as

$S=\sigma_{\gamma} p L$ where $\sigma_{\gamma}$ is the shear strength of the metal, $L$ is length of the pore, and $p$ is the perimeter of the failure surface. If the extrusion is cylindrical, $p$ equals $\pi\left(d_{p}-2 t\right)$. 
The force equilibrium during the intrusion process can be estimated based on Washburn's equation (Washburn 1921) as

$P_{L}-P_{G}=-\frac{4 \gamma \cos \theta}{d_{p}}$

where $P_{L}$ is the driven pressure of the intrusion, $P_{G}$ is the pressure of gas in the pore, $\gamma$ is the surface tension of the liquid, and $\theta$ is the contact angle. The pressure of the gas $P_{G}$ can be estimated based on Washburn's equation and Boyle's law, that in this case can be written as

$P_{A} V_{i}=P_{G} V_{f}$

where $P_{A}$ is the atmosphere pressure, $V_{i}$ and $V_{f}$ are the volume of the gas in the pore before and after the intrusion respectively. It is shown that for pores with $d_{p}$ greater than 3 um, $P_{G} / P_{L}$ is greater than $95 \%$ and $V_{f} / V_{i}$ is less than $1 \%$ when $P_{L}=12.4 \mathrm{MPa}$. For such pores, therefore, one can assume that $P_{G}=$ $P_{L}=12.4 \mathrm{MPa}$ and $V_{f}$ is negligible. The criterion for shear failure in the metal can be found by making $N_{e x}=S$ and $P_{G}=12.4$, giving

$\frac{L}{d_{p}-2 t}<\frac{12.4 \mathrm{MPa}}{4 \sigma_{\gamma}}$

It can be seen from Eq. (6) that wide and shallow pores can be more easily extruded. Based on Table $1, \sigma_{\gamma}$ of Wood's metal and Field's metal can be assume to be around 60 and $30 \mathrm{MPa}$ respectively. This indicates that only very shallow pores with $L / d_{p}-2 t<0.1$ may be subject to shear failure. Such pores may easily be removed during polishing of the sample and thus would not be visible in BSE observation. Thus, the calculations here indicate that the observed extrusion phenomenon is not due to shear failure of the metal.

Therefore, bonding failure is the preferred failure mode. The bonding resistance $\mathrm{R}$ is given as

$R=\tau p L$

where $\tau$ is the interfacial shear strength between the metal and the matrix. The failure criterion is obtained by making $N_{e x}=S$ and $P_{G}=12.4$, giving

$\frac{L}{d_{p}}<\frac{12.4 \mathrm{Mpa}}{4 \tau}$
From observation of extruded metal strips, the maximum $L / d_{p}$ observed is around 15 , giving an estimation of $\tau=0.2 \mathrm{MPa}$. This indicates that Field's metal interacts very weakly with the cement matrix when hardened. In contrast, Wood's metal tends to have very strong bond resistance with the cement matrix, preventing the extrusion phenomenon. Using $\mathrm{Eq}(8), \tau$ needs to be greater than around $3.1 \mathrm{MPa}$ to resist the extrusion of pores with $L / d_{p}>1$.

From our elaboration of the mechanism of extrusion, we conclude that extrusion is not due to the lower strength of Field's metal but due to the weak bonding between Field's metal and the cement matrix. This finding indicates that intrusion and extrusion using Field's metal may induce less damage to pores due to the advance and withdrawal of metal. For that reason, Field's metal is a good candidate for studying the hysteresis of the intrusion/extrusion process. It should be noted that, the intrusion/extrusion of low melting point metal and mercury (Willis et al. 1998) may alter some characteristics of the OPC. Despite the darkening effect on the samples (Diamond 2000), the knowledge about the effect of intrusion/extrusion is still very limited. Further study is essential for a comprehensive understanding on such effect.

\section{Conclusions}

The results demonstrate that centrifugation is a safe and effective method for providing and controlling pressure of melted liquid alloys for intrusion into cementitious samples. The BSE image of the processed sample demonstrates high resolution and contrast of pores, with an observation range from around $100 \mathrm{~nm}$ to $10 \mu \mathrm{m}$. The results suggests that the intrusion pressure of around $12 \mathrm{MPa}$ is adequate to fill pores for BSE imaging. Almost identical cumulative pore diameter distribution curves were found for Field's metal and Wood's metal intruded samples between $100 \mathrm{~nm}$ and $10 \mu \mathrm{m}$, indicating that Field's metal can replace Wood's metal for observation of pores in this range. For pores $>10 \mu \mathrm{m}$, the phenomenon of extrusion is observed with Field's metal, but can be eliminated by pre-vacuuming the sample. Analysis of the extrusion mechanism indicates that it is attributable to the weak bonding between the Field's 
metal and the cement matrix. The new scheme showed promising potential in characterization of geomechanical materials. Future study will involve the extension of the CLMI method to rocks, sand and clay.

Acknowledgments The authors are grateful for the financial support of the Australian Research Council in conducting this study. The authors acknowledge the use of facilities within the Monash Centre for Electron Microscopy.

\section{References}

Abell AB, Willis KL, Lange DA (1999) Mercury intrusion porosimetry and image analysis of cement-based materials. J Colloid Interface Sci 211(1):39-44

Standards Australia (2010) Australian Standard AS 3972-2010: general purpose and blended cement. Standards Australia, Sydney

Chen R, Whanger P, Weswig P (1975) Selenium-induced redistribution of cadmium binding to tissue proteins: a possible mechanism of protection against cadmium toxicity. Bioinorg Chem 4(2):125-133

Diamond S (2000) Mercury porosimetry: an inappropriate method for the measurement of pore size distributions in cementbased materials. Cem Concr Res 30(10):1517-1525

Dullien FAL (1981) Wood's metal porosimetry and its relation to mercury porosimetry. Powder Technol 29(1):109-116

Ferreira T, Rasband W (2011) The ImageJ user guide. National Institutes of Health, Bethesda

Kim D-G, Dong XN, Cao T, Baker KC, Shaffer RR, Fyhrie DP, Yeni YN (2006) Evaluation of filler materials used for uniform load distribution at boundaries during structural biomechanical testing of whole vertebrae. J Biomech Eng 128(1):161-165

Kjellsen K, Monsøy A, Isachsen K, Detwiler R (2003) Preparation of flat-polished specimens for SEM-backscattered electron imaging and X-ray microanalysis-importance of epoxy impregnation. Cem Concr Res 33(4):611-616

Kumar R, Bhattacharjee B (2003) Study on some factors affecting the results in the use of MIP method in concrete research. Cem Concr Res 33(3):417-424
Lange DA, Jennings HM, Shah SP (1994) Image analysis techniques for characterization of pore structure of cementbased materials. Cem Concr Res 24(5):841-853

Lloyd GE (1987) Atomic number and crystallographic contrast images with the SEM: a review of backscattered electron techniques. Mineral Mag 51(359):3-19

Lloyd RR, Provis JL, Smeaton KJ, van Deventer JS (2009) Spatial distribution of pores in fly ash-based inorganic polymer gels visualised by wood's metal intrusion. Microporous Mesoporous Mater 126(1):32-39

MatWeb L (2015) Bi50-Pb26.7-Sn13.3-Cd10 Bismuth-LeadTin-Cadmium Fusible Alloy, MatWeb material property database

MatWeb L (2015) Indium Corp. Indalloy ${ }^{\circledR} 19$ In-Bi-Sn Fusible Alloy. MatWeb material property database

Moro F, Böhni H (2002) “Ink-Bottle effect in mercury intrusion porosimetry of cement-based materials: volume 246, number 1 (2001), pages 135-149. J Colloid Interface Sci 249(1):262

Nemati K (2000) Preserving microstructure of concrete under load using the Wood's metal technique. Int J Rock Mech Min Sci 37(1):133-142

Ridler T, Calvard S (1978) Picture thresholding using an iterative selection method. IEEE Trans Syst Man Cybern 8(8):630-632

Schubauer-Berigan MK, Dierkes JR, Monson PD, Ankley GT (1993) $\mathrm{pH}$-dependent toxicity of $\mathrm{Cd}, \mathrm{Cu}, \mathrm{Ni}, \mathrm{Pb}$ and $\mathrm{Zn}$ to Ceriodaphnia dubia, Pimephales promelas, Hyalella azteca and Lumbriculus variegatus. Environ Toxicol Chem 12(7):1261-1266

Stroeven P, Hu J, Koleva D (2010) Concrete porosimetry: aspects of feasibility, reliability and economy. Cem Concr Compos 32(4):291-299

Washburn EW (1921) Note on a method of determining the distribution of pore sizes in a porous material. Proc Natl Acad Sci 7:115

Willis KL, Abell AB, Lange DA (1998) Image-based characterization of cement pore structure using wood's metal intrusion. Cem Concr Res 28(12):1695-1705

Wong H, Head M, Buenfeld N (2006) Pore segmentation of cement-based materials from backscattered electron images. Cem Concr Res 36(6):1083-1090 